\title{
Risk evaluation of abdominal aortic aneurysms based on both sex and morphology
}

\author{
An-Qiang Sun ${ }^{1, \#}$, Ya-Xing Zhang ${ }^{1, \#, ~ Z a i-P i n ~ X u ², ~ S h u-Q i ~ R e n ', ~ X i a o-Y a n ~ D e n g 1 ', ~ R o b e r t ~ G u i d o i n ~}{ }^{1,3}$ \\ 'Beijing Advanced Innovation Center for Biomedical Engineering\&Key Laboratory for Biomechanics and Mechanobiology of \\ Ministry of Education, School of Biological Science and Medical Engineering, Beihang University, Beijing 100083, China. \\ ${ }^{2}$ College of Animal Science, Guizhou University, Guiyang 550025, Guizhou, China. \\ ${ }^{3}$ Department of Surgery, Faculty of Medicine, Université Laval and CHU de Québec Research Centre, Québec, QC Canada G1A \\ OA6, Canada. \\ \#Authors contributed equally.
}

Correspondence to: Prof. Xiao-Yan Deng, Key Laboratory for Biomechanics and Mechanobiology of Ministry of Education, School of Biological Science and Medical Engineering, Beihang University, Beijing 100083, China. E-mail: dengxy1953@buaa.edu. cn; Prof. Robert Guidoin, Department of Surgery, Faculty of Medicine, Université Laval and CHU de Québec Research Centre, Québec, QC Canada G1A 0A6, Canada. E-mail: robert.guidoin@fmed.ulaval.ca

How to cite this article: Sun $A Q$, Zhang $Y X, X u Z P$, Ren $S Q$, Deng $X Y$, Guidoin R. Risk Evaluation of abdominal aortic aneurysms based on both sex and morphology. Vesse/ P/us 2020;4:13. http://dx.doi.org/10.20517/2574-1209.2020.02

Received: 3 Jan 2020 First Decision: 21 Jan 2020 Revised: 26 Feb 2020 Accepted: 19 Mar 2020 Published: 16 May 2020

Science Editor: Cristiano Spadaccio Copy Editor: Jing-Wen Zhang Production Editor: Jing Yu

\begin{abstract}
Aim: To predict the peak wall stress in abdominal aortic aneurysms (AAAs) considering both morphological factors (maximum diameter, asymmetry index, and wall thickness) and sex differences, in order to assess the risk of AAA rupture more accurately.
\end{abstract}

Methods: Basic models of AAA focusing on different sexes with a range of morphological parameters were constructed. Using the Design-expert software for three-factor response surface methodology, 20 experimental models were built as well with the SolidWorks software. Fluid-structure interaction analysis was used to obtain stress distribution along the AAA wall. Polynomial regression equations were fitted to peak stresses in all experimental models.

Results: Based on fluid-structure interaction simulation data in the nonlinear polynomial regression model, separate equations for peak wall stress in AAA with regard to males and females were obtained. Morphological factors and sex differences have significant influence on peak wall stress. In some models, even when the maximum AAA diameter was relatively small, the peak wall stress became high. For the same maximal transverse measurement, when the AAA wall was thin and the asymmetry index large, or the former was thick and the latter small, the peak

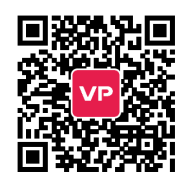


wall stress observed in males was higher than that in females.

Conclusion: To evaluate the risk of rupture of AAA more precisely and specifically, the present study proposes a new prediction method (based on equations) that includes more indicators such as sex and morphology, based on numerical biomechanical simulations, which were confirmed as such. This study provides a sex-specific clinical reference to assess the aforementioned risk of AAA rupture.

Keywords: Abdominal aortic aneurysm, sex difference, morphology, peak stress, rupture risk assessment

\section{INTRODUCTION}

Abdominal aortic aneurysms (AAAs) are a high-risk vascular disease characterised by local expansion of the abdominal aorta to more than $50 \%$ of its original diameter. If AAAs are not treated in time, it is likely to expand and rupture eventually ${ }^{[1]}$. Its prevalence in individuals older than 60 years is at least four times higher in men than women ${ }^{[2]}$. The mortality rate associated with a ruptured AAA is as high as $60 \%-80 \%{ }^{[3]}$ and about 15,000 such deaths occur in the United States every year ${ }^{[4]}$. Clinically, the treatment for AAA includes open repair and endovascular aneurysm repair.

Previously, indications for surgical treatment of AAA were based on the largest vessel diameter (greater than 5.5 or $5 \mathrm{~cm})^{[5]}$. However, relying mainly on maximal transverse measurement as a criterion to determine whether an AAA should be treated may delay the optimal timing for intervention in some patients or even lead to serious consequences such as rupture. In recent years, other biomechanical risk factors for AAA rupture have been used to more accurately predict its development, such as morphological factors (maximum diameter, asymmetry index, wall thickness, etc. ${ }^{[6,7]}$. Vorp et al. ${ }^{[8]}$ established a threedimensional model of an AAA and found that an aneurysm which was asymmetric had a great influence on the distribution of wall stress, and peak wall stress increased nonlinearly with greater asymmetry. From a biomechanical point of view, a ruptured AAA occurs when local stress of the vascular wall exceeds the mechanical strength of its material. Thus, the higher local stress is along the AAA's wall, the higher the risk of a ruptured $\mathrm{AAA}^{[9]}$. Besides peak wall stress, blood flow rheology has also been considered. For example, the wall shear stress induced by pulsatile flow, due to friction between blood flow and the inner wall of an AAA, was found to influence the rupture risk and function of AAA by damaging the endothelium and inducing AAA wall remodelling ${ }^{[10-13]}$.

Recent studies have found that the risk of AAA rupture has significant sex differences. The UK Small Aneurysm Trial, after a 10-year (1991-2001) follow-up of 496 patients, found that 5\% of 411 males died of a ruptured AAA, while $14 \%$ of 85 females did, which suggests that the risk of rupture in affected women was three to four times higher ${ }^{[14]}$. The medical literature has also reported a myriad of publications about the importance of sex differences ${ }^{[15-18]}$ with poorer outcomes in women. This observation has also been confirmed in a large number of observational studies and randomized controlled ones in recent years ${ }^{[19,20]}$. As noted by Ash et al. ${ }^{[21]}$, there has been significant debate among vascular specialists regarding AAA between men and women. A much higher incidence is seen in men, with a male to female ratio of $4: 1^{[22]}$. Women though, are older at presentation, exhibit faster rates of AAA growth and thus have a higher risk of rupture at lower diameters ${ }^{[23]}$. As the debate surrounding the definition, diagnosis and treatment of AAA in women continues, more specific guidelines are needed ${ }^{[24]}$.

The mechanism underlying sex-dependent differences in AAA rupture is still unclear however. The reason may be due to women having an aorta diameter that is generally smaller than in men. Therefore, for similarly sized AAAs, the degree of expansion in females is greater, and consequently, the risk of rupture increases accordingly, which suggests that the threshold for treatment of the maximum diameter in female 


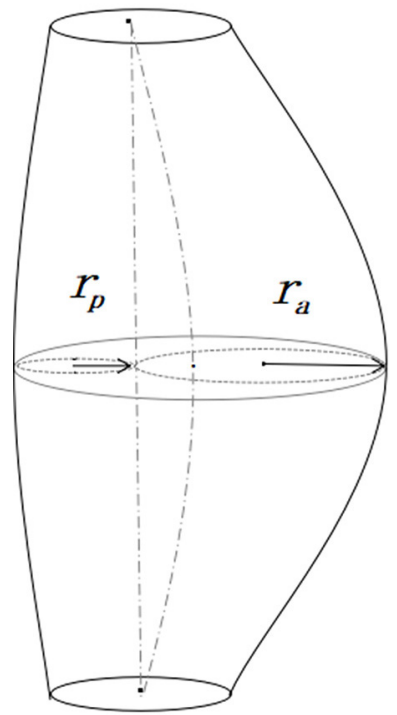

Figure 1. Three-dimensional model of abdominal aortic aneurysm. $r_{\mathrm{p}}$ : maximum posterior radius; $r_{\mathrm{a}}$ : maximum anterior radius

AAA patients should be reduced ${ }^{[25,26]}$.

In the present study, a series of quantitative simulations were conducted to investigate the relationships between the risk of a AAA rupture and patient sex and AAA morphology parameters. Based on these simulation results, equations for the peak wall stress for male and female patients were derived. A threedimensional grid cube representing the risk comparison between both sexes has also been built considering wall thickness, asymmetry and maximum diameters of an AAA.

\section{METHODS}

\section{Design of experiments}

Based on the central composite design in response surface methodology, the maximum diameter, asymmetry index and wall thickness of AAA were selected as independent variables, and the peak wall stress measurement was used as the response value ${ }^{[27-29]}$. Firstly, the morphology parameters for AAA in males and females over 70 years of age were determined according to clinical studies found in the literature ${ }^{[30]}$. Measurements of the diameter in the upper and lower parts of the AAA were 2.8 and $2.3 \mathrm{~cm}$ for males, and 2.7 and $2 \mathrm{~cm}$ respectively for females. The length of the abdominal aorta was set at $12 \mathrm{~cm}$ for both sexes. The maximum diameters in AAA ranged from 3 to $6 \mathrm{~cm}$ for males and 2.7 to $5 \mathrm{~cm}$ for females. As shown in Figure 1, the asymmetry index $\beta$ in AAA is defined as the ratio of the maximum posterior radius $r_{p}$ to the maximum anterior radius $r_{a}\left(\beta=r_{p} / r_{a}\right)$, which is identified to range from 0.3 to 1 . Previous studies have revealed that the average wall thickness in AAA is generally $1.5 \mathrm{~mm}^{[31]}$. Therefore, its variation range in the test design was set to $0.8 \mathrm{~mm}$ to $2.2 \mathrm{~mm}$.

To define experimental groups, we selected a two-factor central composite design for this design as an example. Figure 2 represents the test points during a setup for a two-factor universal spiral combination design. For a full factorial one with $p=3$ variables, there were total $m_{c}=2^{p}=8$ points in this work, including two test points of $\pm \mathrm{r}$ on each axis, so their $\mathrm{m}_{\mathrm{r}}=2^{\mathrm{p}}=6$. As for $\mathrm{m}_{0}$, it was defined as how often the centre point test in which each factor takes a zero level ${ }^{[32]}$. Therefore, the overall number of trials was $\mathrm{m}=$ $\mathrm{m}_{\mathrm{c}}+\mathrm{m}_{\mathrm{r}}+\mathrm{m}_{\mathrm{o}}$.

Finally, for the experiment design in this study, the coding factors of the models for males and females and 


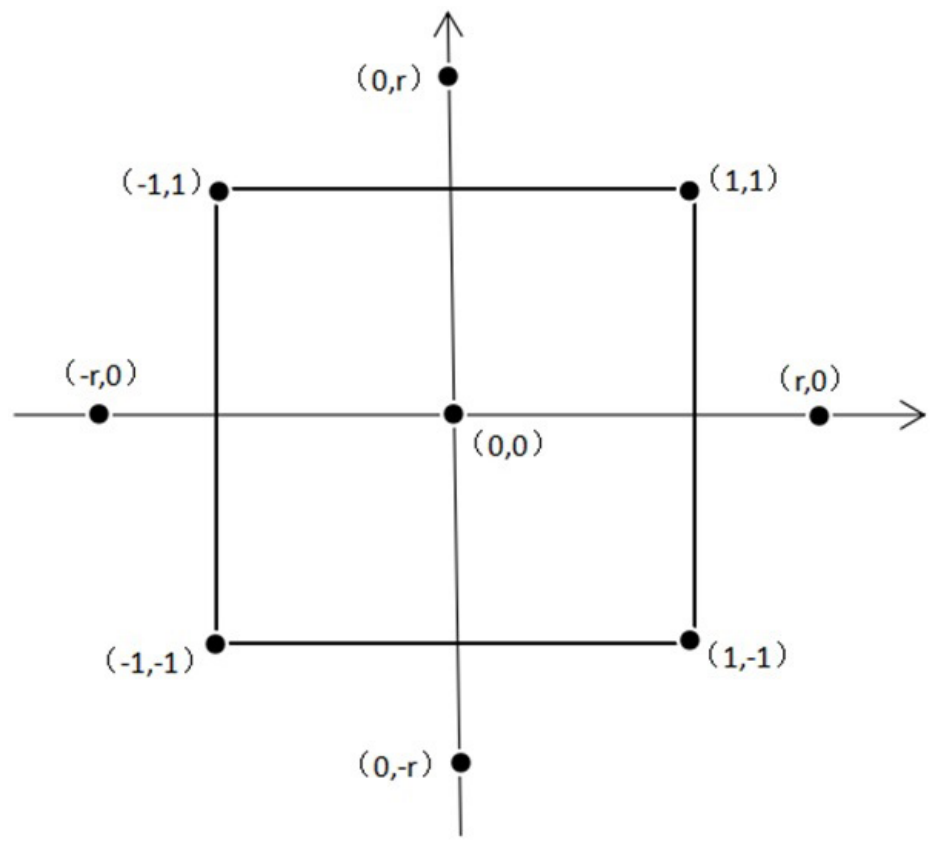

Figure 2. Distribution of test points for a two-factor general rotating combination design

Table 1. Coding factors for abdominal aortic aneurysm models designed for males and females and corresponding values

\begin{tabular}{llcllll}
\hline \multirow{2}{*}{ Factors } & & \multicolumn{3}{c}{ Coded levels } \\
\cline { 2 - 6 } & & $\mathbf{- r}(\mathbf{- 1 . 6 8 2 )}$ & $\mathbf{- 1}$ & $\mathbf{0}$ & $\mathbf{+ 1}$ & $\mathbf{+ r}(\mathbf{1 . 6 8 2})$ \\
\hline Diameter $(\mathrm{mm})$ & Male & 30 & 36.1 & 45 & 53.9 & 60 \\
& Female & 27 & 31.7 & 38.5 & 45.3 & 50 \\
Asymmetry index & 0.3 & 0.44 & 0.65 & 0.86 & 1 \\
Wall thickness (mm) & 0.8 & 1.08 & 1.5 & 1.92 & 2.2 \\
\hline
\end{tabular}

Table 2. Design matrix as well as simulated and predicted peak wall stresses for the abdominal aortic aneurysm models designed for males

\begin{tabular}{lccccc}
\hline $\begin{array}{l}\text { Test model } \\
(\mathbf{m a l e})\end{array}$ & $\begin{array}{c}\text { Maximum diameter } \\
\mathbf{D}_{\text {max }}(\mathbf{m m})\end{array}$ & Asymmetry index $\beta$ & Wall thickness t (mm) & $\begin{array}{c}\text { Simulated peak wall } \\
\text { stress (KPa) }\end{array}$ & $\begin{array}{c}\text { Predicted peak wall } \\
\text { stress }(\mathbf{K P a})\end{array}$ \\
\hline 1 & $1(53.9)$ & $1(0.86)$ & $1(1.92)$ & 273.85 & 276.29 \\
2 & $1(53.9)$ & $1(0.86)$ & $-1(1.08)$ & 531.13 & 533.65 \\
3 & $1(53.9)$ & $-1(0.44)$ & $1(1.92)$ & 415.19 & 415.67 \\
4 & $1(53.9)$ & $-1(0.44)$ & $-1(1.08)$ & 822.74 & 823.11 \\
5 & $-1(36.1)$ & $1(0.86)$ & $1(1.92)$ & 167.33 & 178.41 \\
6 & $-1(36.1)$ & $1(0.86)$ & $-1(1.08)$ & 326.61 & 337.61 \\
7 & $-1(36.1)$ & $-1(0.44)$ & $1(1.92)$ & 267.29 & 276.23 \\
8 & $-1(36.1)$ & $-1(0.44)$ & $-1(1.08)$ & 576.48 & 585.51 \\
9 & $r(60)$ & $0(0.65)$ & $0(1.5)$ & 487.86 & 489.81 \\
10 & $-r(30)$ & $0(0.65)$ & $0(1.5)$ & 225.48 & 207.67 \\
11 & $0(45)$ & $r(1)$ & $0(1.5)$ & 257.02 & 245.41 \\
12 & $0(45)$ & $-r(0.3)$ & $0(1.5)$ & 574.79 & 571.11 \\
13 & $0(45)$ & $0(0.65)$ & $r(2.2)$ & 228.5 & 220.15 \\
14 & $0(45)$ & $0(0.65)$ & $-r(0.8)$ & 820.63 & 817.73 \\
15 & $0(45)$ & $0(0.65)$ & $0(1.5)$ & 374.61 & 375.67 \\
16 & $0(45)$ & $0(0.65)$ & $0(1.5)$ & 374.73 & 375.67 \\
17 & $0(45)$ & $0(0.65)$ & $0(1.5)$ & 371.05 & 375.67 \\
18 & $0(45)$ & $0(0.65)$ & $0(1.5)$ & 372.17 & 375.67 \\
19 & $0(45)$ & $0(0.65)$ & $0(1.5)$ & 378.92 & 375.67 \\
20 & $0(45)$ & $0(0.65)$ & $0(1.5)$ & 379.23 & 375.67 \\
\hline
\end{tabular}


Table 3. Design matrix as well as simulated and predicted peak wall stresses for the abdominal aortic aneurysm models designed for females

\begin{tabular}{lccccc}
\hline $\begin{array}{l}\text { Test model } \\
\text { (female) }\end{array}$ & $\begin{array}{c}\text { Maximum diameter } \\
\mathbf{D}_{\mathbf{m a x}}(\mathbf{m m})\end{array}$ & Asymmetry index $\beta$ & Wall thickness t (mm) & $\begin{array}{c}\text { Simulated peak wall } \\
\text { stress (KPa) }\end{array}$ & $\begin{array}{c}\text { Predicted peak wall } \\
\text { stress (KPa) }\end{array}$ \\
\hline 1 & $1(45.3)$ & $1(0.86)$ & $1(1.92)$ & 222.16 & 226.76 \\
2 & $1(45.3)$ & $1(0.86)$ & $-1(1.08)$ & 455.46 & 460.90 \\
3 & $1(45.3)$ & $-1(0.44)$ & $1(1.92)$ & 352.66 & 355.21 \\
4 & $1(45.3)$ & $-1(0.44)$ & $-1(1.08)$ & 719.4 & 721.11 \\
5 & $-1(31.7)$ & $1(0.86)$ & $1(1.92)$ & 146.29 & 151.73 \\
6 & $-1(31.7)$ & $1(0.86)$ & $-1(1.08)$ & 286.3 & 290.90 \\
7 & $-1(31.7)$ & $-1(0.44)$ & $1(1.92)$ & 234.01 & 235.72 \\
8 & $-1(31.7)$ & $-1(0.44)$ & $-1(1.08)$ & 504.1 & 506.65 \\
9 & $r(50)$ & $0(0.65)$ & $0(1.5)$ & 416.54 & 411.55 \\
10 & $-r(27)$ & $0(0.65)$ & $0(1.5)$ & 203.25 & 198.25 \\
11 & $0(38.5)$ & $r(1)$ & $0(1.5)$ & 218.87 & 210.26 \\
12 & $0(38.5)$ & $-r(0.3)$ & $0(1.5)$ & 498.78 & 497.10 \\
13 & $0(38.5)$ & $0(0.65)$ & $r(2.2)$ & 191.94 & 186.79 \\
14 & $0(38.5)$ & $0(0.65)$ & $-r(0.8)$ & 689.14 & 684.00 \\
15 & $0(38.5)$ & $0(0.65)$ & $0(1.5)$ & 315.72 & 316.63 \\
16 & $0(38.5)$ & $0(0.65)$ & $0(1.5)$ & 315.86 & 316.63 \\
17 & $0(38.5)$ & $0(0.65)$ & $0(1.5)$ & 312.13 & 316.63 \\
18 & $0(38.5)$ & $0(0.65)$ & $0(1.5)$ & 314.27 & 316.63 \\
19 & $0(38.5)$ & $0(0.65)$ & $0(1.5)$ & 318.85 & 316.63 \\
20 & $0(38.5)$ & $0(0.65)$ & $0(1.5)$ & 319.94 & 316.63
\end{tabular}

their values are defined in Table 1. Accordingly, 20 model parameters were generated for men and women, respectively (columns $1-4$ of Tables 2 and 3 ).

\section{Geometric model}

All geometric models in this study were built using a three-dimensional modelling software SolidWorks 2016 (Dassault Systèmes SolidWorks Corporation, Waltham, MA, USA) based on the parameters listed in Tables 2 and 3. In order to obtain relatively smooth blood flow, the diameter at the upper and lower parts of the model were extended by 1.5 times. The schematic diagrams of all AAA designs are shown in Figure 3.

\section{Governing equations, boundary conditions and material properties}

The fluid-solid coupling analysis was carried out with ANSYS Workbench 15.0 (Ansys, Inc., Lebanon, NH, USA). The pressure distribution obtained by the Fluid Flow calculation was transmitted to the vascular wall through a fluid-solid coupling system, and the solid structure was determined by using the Static Structural Analysis module to figure out how stress is distributed in the final vascular wall. The fluid-filled domain was stitched using tetrahedral meshes. In order to ensure convergence of the physical quantities calculated iteratively such as velocity and force on the fluid-solid coupling surface, the vascular wall was also stitched as above.

Numerical simulation calculations were based on the Navier-Stokes equation and the mass conservation continuity one, neglecting gravity force:

$$
\begin{aligned}
& \rho(\vec{u} \cdot \nabla) \vec{u}+\nabla p-\mu \Delta \vec{u}=0 \\
& \nabla \cdot \vec{u}=0
\end{aligned}
$$

where, $u$ and $p$ represent the velocity vector $(\mathrm{m} / \mathrm{s})$ and pressure $(\mathrm{Pa})$ respectively, and $\rho$ and $\mu$ are the density $\left(\mathrm{kg} / \mathrm{cm}^{3}\right)$ and viscosity (Pa.s) of the blood.

In order to facilitate convergence of the fluid-solid coupling calculation, a steady-flow analysis was used. 


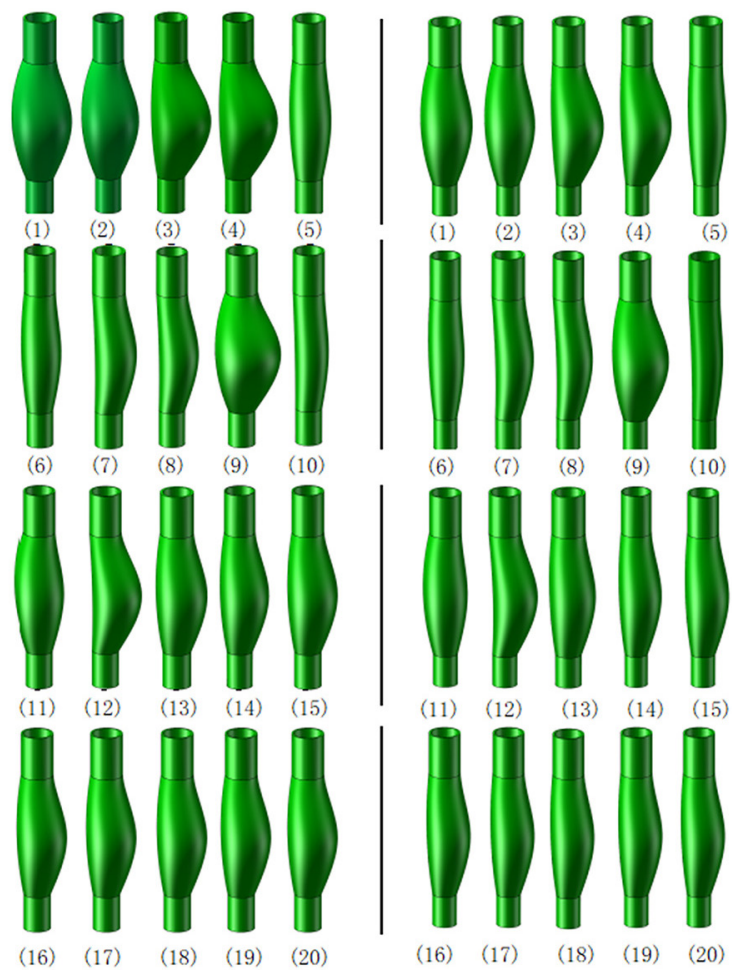

Figure 3. All geometric models (left: male; right: female, the number under each model represents the Test model number from Columns 1 of Tables 2 and 3)

Blood was assumed to be a uniform, continuous, isotropic, incompressible Newtonian fluid with a density value of $1050 \mathrm{~kg} / \mathrm{m}^{3}$ and a viscosity of $0.0035 \mathrm{~Pa} \cdot \mathrm{s}$. For boundary conditions, the inlet flow rate and the outlet pressure were fitted to the simulation with reference to the literature ${ }^{[33]}$. The outlet pressure of models for males and females was equivalent to that of systolic blood at 16,414 $\mathrm{Pa}(\sim 123 \mathrm{mmHg})$, and the corresponding inlet flow velocity were $0.1614 \mathrm{~m} / \mathrm{s}$ for men and $0.1797 \mathrm{~m} / \mathrm{s}$ for women. The CFD software package which consisted of ANSYS Fluent 15.0 was used for simulation, and the SIMPLE algorithm was applied.

The wall of the AAA was assumed as a linear, isotropic elastic material. According to previous studies, the stiffness of the blood vessel wall in males is higher than that in females ${ }^{[34,35]}$. Therefore, the Young's modulus obtained for women in this study was defined as $80 \%$ of men. Finally, the density was set to $2000 \mathrm{~kg} / \mathrm{m}^{3}$, the Poisson's ratio 0.45 , and the Young's modulus was $2.7 \mathrm{MPa}$ for men and $2.16 \mathrm{MPa}$ for women ${ }^{[36]}$. Displacements along all directions at two ends were constrained.

After all the simulations, a multivariate regression analysis will be performed on the peak Von Mises stress values of the test models using Design-Expert software Version 10 to obtain cubic polynomial equations to predict the related stresses.

\section{RESULTS}

The simulated peak wall stress of each model has been shown in Table 2 and Table 3 (the penultimate column).

Two models with significant differences in the maximum diameter of AAA for the male group were selected, and the stress distribution contours are shown in Figure 4. Although the maximum diameter measured for model 2 was higher than that of model $8(5.39 \mathrm{~cm} v s .3 .61 \mathrm{~cm})$, the peak wall stress of model 

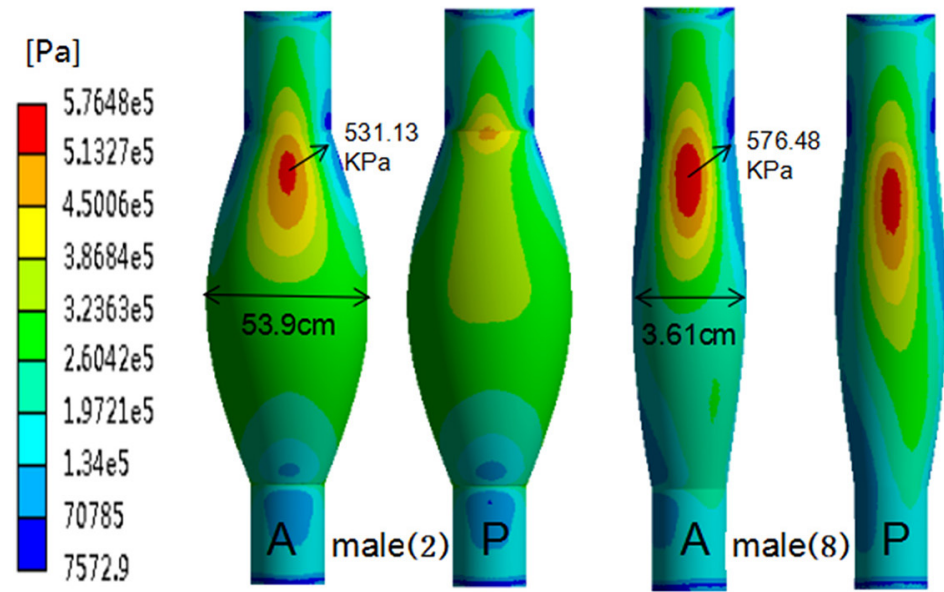

Figure 4. Stress distribution in models 2 and 8 of the male group. A: anterior; P: posterior
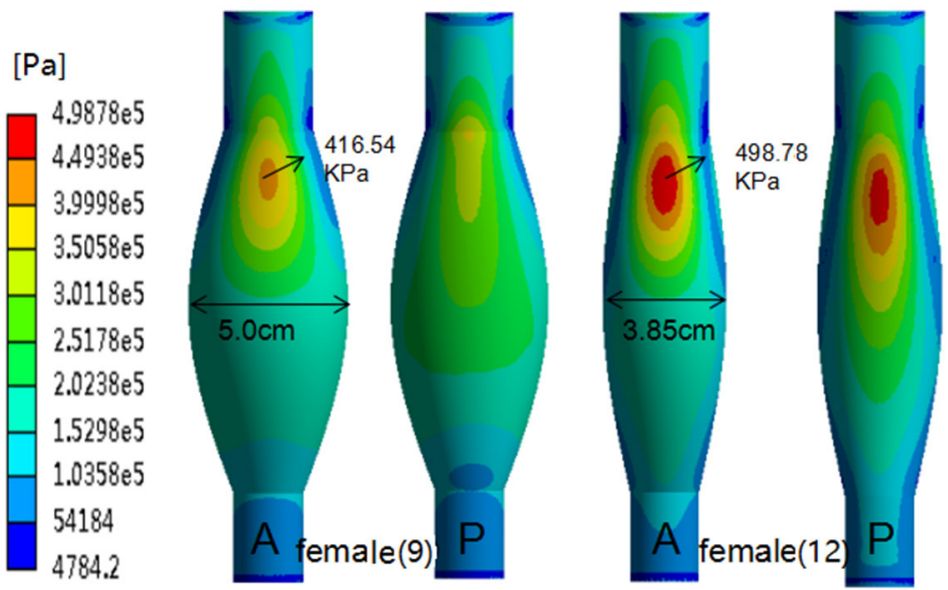

Figure 5. Stress distribution in models 9 and 12 of the female group.A: anterior; P: posterior

2 remained lower than that of model $8(531.13 \mathrm{KPa} v s .576 .48 \mathrm{KPa})$. Similar results were observed when model 2 was compared with models 9,12 , and 14 , and when model 1 was compared with models $6,8,12$, 14, and 15. In the female testing group, the stress distribution contours of models 9 and 12 are presented in Figure 5. The maximum diameter measured for the model 9 was higher than that of model $12(5 \mathrm{~cm}$ vs. $3.85 \mathrm{~cm})$, but the peak wall stress of model 9 turned out lower than that of model $12(416.54 \mathrm{KPa} v s$. $498.78 \mathrm{KPa}$ ). This situation was also not unique in the female group, when model 9 was compared with models 2, 4, and 14. Comparison of results obtained for stress distribution showed that using only the maximum diameter to predict the risk of AAA rupture was not enough and might lead to non-optimal decisions.

Based on all simulation results, a multivariate regression analysis was performed on the peak Von Mises stress values of the test models in Tables 2 and 3 using Design-Expert software Version 10 to obtain cubic polynomial equations to predict the related stresses:

$$
\begin{aligned}
\text { Male_PWS }= & 375.67+83.87 \mathrm{~d}-96.82 \mathrm{a}-177.64 \mathrm{t}-10.39 \mathrm{ad}-24.54 \mathrm{dt} \\
& +37.52 \mathrm{at}-9.52 \mathrm{~d}^{2}+11.52 \mathrm{a}^{2}+50.64 \mathrm{t}^{2}+35.98 \mathrm{~d}^{2} \mathrm{t}
\end{aligned}
$$

Female_PWS $=316.63+63.06 \mathrm{~d}-86.05 \mathrm{a}-149.16 \mathrm{t}-11.11 \mathrm{ad}-23.74 \mathrm{dt}$

$$
+32.94 \mathrm{at}-4.1 \mathrm{~d}^{2}+13.34 \mathrm{a}^{2}+42.76 \mathrm{t}^{2}+22.89 \mathrm{~d}^{2} \mathrm{t}+9.31 \mathrm{a}^{2} d
$$

where $a, d, t$ are coded dimensionless variables of the asymmetry index, the maximum diameter of the 
Table 4. Analysis of variance for fit regression regarding male function

\begin{tabular}{llllll}
\hline Source & Seq SS & DF & MS & F value & P value \\
\hline Model & $615,971.1$ & 10 & $61,597.11$ & 505.79 & $<0.0001$ \\
A-Diameter & $96,231.85$ & 1 & $96,231.85$ & 790.18 & $<0.0001$ \\
B-Asymmetry & 127,100 & 1 & 127,100 & 1043.33 & $<0.0001$ \\
C-Wall thickness & 175,300 & 1 & 175,300 & 1439.51 & $<0.0001$ \\
AB & 863.62 & 1 & 863.62 & 7.09 & 0.0259 \\
AC & 4819.66 & 1 & 4819.66 & 39.58 & 0.0001 \\
BC & $11,263.50$ & 1 & $11,263.50$ & 92.49 & $<0.0001$ \\
A $^{2}$ & 1318.21 & 1 & 1318.21 & 10.82 & 0.0094 \\
B $^{2}$ & 1859.77 & 1 & 1859.77 & 15.27 & 0.0036 \\
C $^{2}$ & $35,937.61$ & 1 & $35,937.61$ & 295.09 & $<0.0001$ \\
A $^{2}$ C & 4243.63 & 1 & 4243.63 & 34.85 & 0.0002 \\
Residual error & 1096.06 & 9 & 121.78 & & \\
Lack of fit & 1039.04 & 4 & 259.76 & 22.78 & 0.0021 \\
Pure error & 57.01 & 5 & 11.40 & & \\
Total & 617,100 & 19 & & \\
\hline
\end{tabular}

DF: degree of freedom; MS: mean square deviation

Table 5. Analysis of variance for fit regression regarding female function

\begin{tabular}{llllll}
\hline Source & Seq SS & DF & MS & F value & P value \\
\hline Model & 458,400 & 11 & $41,675.00$ & 994.59 & $<0.0001$ \\
A-Diameter & $22,746.31$ & 1 & $22,746.31$ & 542.85 & $<0.0001$ \\
B-Asymmetry & 100,400 & 1 & 100,400 & 2395.54 & $<0.0001$ \\
C-Wall thickness & 123,600 & 1 & 123,600 & 2949.85 & $<0.0001$ \\
AB & 988.35 & 1 & 988.35 & 23.59 & 0.0013 \\
AC & 4509.65 & 1 & 4509.65 & 107.62 & $<0.0001$ \\
BC & 8680.35 & 1 & 8680.35 & 207.16 & $<0.0001$ \\
$A^{2}$ & 247.06 & 1 & 247.06 & 5.90 & 0.0413 \\
$B^{2}$ & 2493.07 & 1 & 2493.07 & 59.50 & $<0.0001$ \\
$C^{2}$ & $25,615.03$ & 1 & $25,615.03$ & 611.31 & $<0.0001$ \\
$A^{2} C$ & 1718.25 & 1 & 1718.25 & 41.01 & 0.0002 \\
AB & 289.27 & 1 & 289.27 & 6.90 & 0.0303 \\
Residual error & 335.21 & 8 & 41.90 & & 14.55 \\
Lack of fit & 300.76 & 3 & 100.25 & & 0.0066 \\
Pure error & 34.45 & 5 & 6.89 & & \\
Total & 458,800 & 19 & & & \\
\hline
\end{tabular}

DF: degree of freedom; MS: mean square deviation

AAA, and wall thickness, respectively.

To verify the two equations for predicting outcomes, the coefficients of determination were obtained: for $R_{M}^{2}=0.9982$ for Male_PWS , and $R_{F}^{2}=0.9993$ for Female_PWS, which indicates that the model is applicable. The analysis of variance [Tables 4 and 5] showed that the three selected factors (diameter, asymmetry and wall thickness) were significant for peak stress $(P<0.05)$. However, the lack of fit in this work was biased towards the other side of statistical significance $(P<0.05)$, because the computation was ideal when simulating a repeating model, instead of true replication of the simulation ${ }^{[21]}$. Using the two predictive equations, the predicted peak stress in each model were obtained and presented in Tables 2 and 3 (the last column). Comparison of the simulated and predicted peak stresses in Tables 2 and 3 reveals good fit of the two regression models.

In Equations 3 and 4, the absolute value of the coefficient for first order from the regression equation reflects the contributions of each single factor to the response value. Then, we can sort out the three factors by their contribution to peak stress: AAA wall thickness, asymmetry index and maximum AAA diameter. 


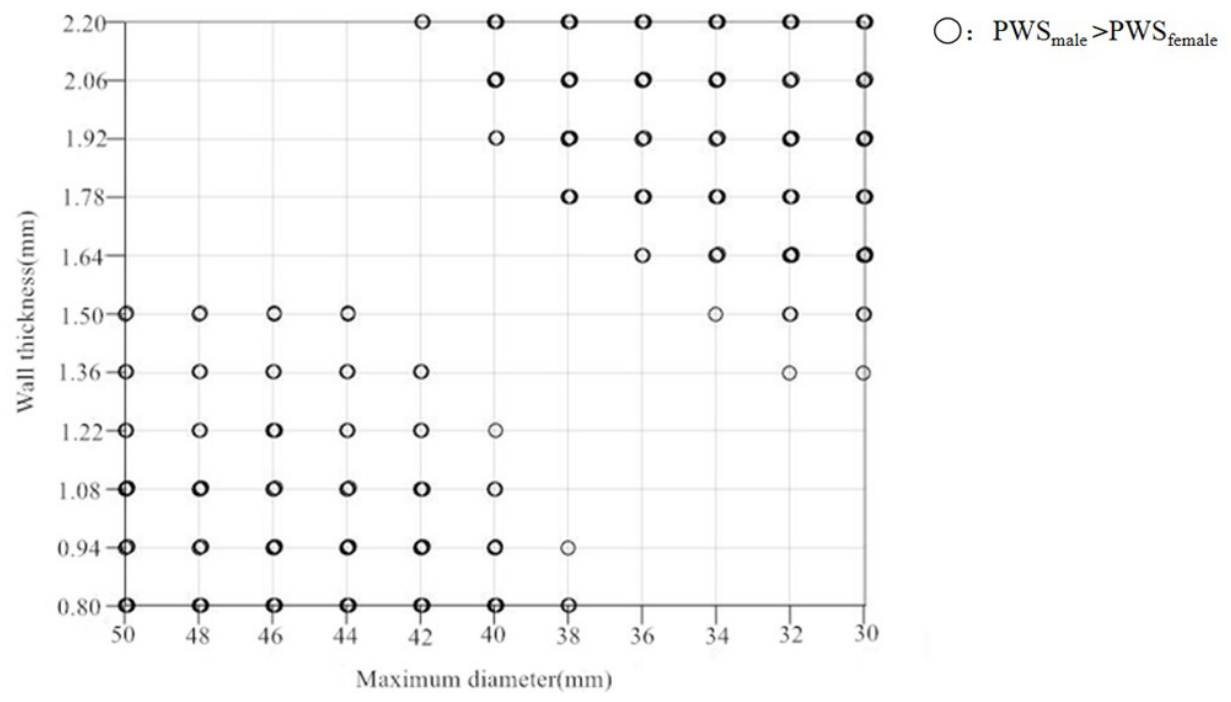

Figure 6. Scatter plot showing the correlation between peak stress and relative magnitude of the wall thickness in males and females with abdominal aortic aneurysm (into the plane of the wall thickness - maximum diameter)

This conclusion was consistent in both types of models for males and females. When the asymmetry index corresponded to 0.44 and the wall thickness to $2 \mathrm{~mm}$, AAAs featuring maximum diameters of $5 \mathrm{~cm}$ for women and $5.5 \mathrm{~cm}$ for men had comparable risk of rupture. Similarly, according to Forbes et al. ${ }^{[20]}$, AAAs with maximal values of $5.2 \mathrm{~cm}$ for females and $5.5 \mathrm{~cm}$ for males presented a comparable risk. However, when the asymmetry index and wall thickness change, the peak stress of the AAA does the same, which means that the conclusions of Forbes et al. ${ }^{[20]}$ are not applicable in all cases ${ }^{[37]}$.

To more accurately reflect the relative magnitude of the risk of rupture in AAA, a range of values were selected for each variable in the models designed for males and females (a maximum diameter from $3 \mathrm{~cm}$ to $5 \mathrm{~cm}$, an asymmetry index from 0.3 to 1 , and a wall thickness from $0.8 \mathrm{~mm}$ to $2.2 \mathrm{~mm}$ ) to determine their intersection. Eleven discrete points were uniformly taken within the variation range of each factor, and the dimensions were unified. Then, a scatter [Figure 6] and a 3D mesh [Figure 7] plots were obtained. As presented in Figure 6, the plot is projected onto the plane of the wall thickness-maximum diameter. It can be seen that the distribution trend of the points is obvious and there was no scatter in space. For AAAs featuring the same maximum diameter, $\mathrm{PWS}_{\text {male }}>\mathrm{PWS}_{\text {female }}$ occurred when the vascular wall was thin and the asymmetry index was large, or when the former was thick and the latter was small.

\section{DISCUSSION}

Traditionally, the maximum diameter of AAA has generally been used as a criteria for surgical treatment. Increasingly however, clinical studies have suggested a deficiency in this maximal transverse measurement, with greater consideration of morphology parameters in risk assessment of AAA over the last decades. In recent years, studies have revealed that male and female differences were also important and should be evaluated ${ }^{[1,20,25]}$. In the present study, both sex differences and morphological factors were considered concurrently in predicting AAA rupture in high-risk patients. Nevertheless, it is of paramount importance to include biomechanical assessment of the structures involved in AAA for a more thorough evaluation. Collagen composition in the wall of AAAs in men and women are similar exception for its cross-linking ${ }^{[38]}$ but the risk of rupture is multifactorial from a biomechanical point of view ${ }^{[39,40]}$. Sex affects AAA formation but the role of hormones is still poorly understood.

Based on a series of numerical FSI simulations that considered patient sex and morphological factors 


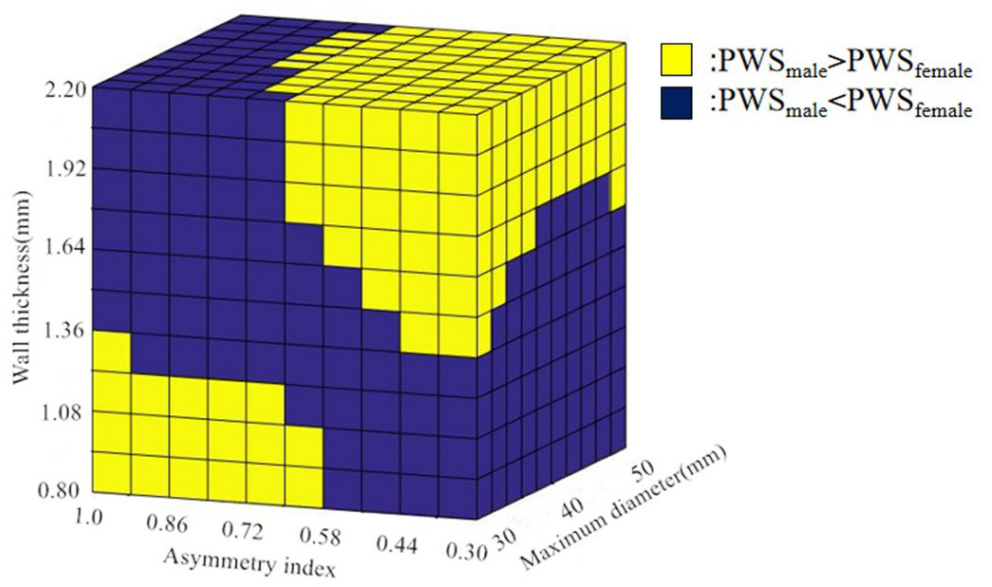

Figure 7. 3D grid cube presenting the relationship between peak wall stresses in males and females

(including maximum diameter, asymmetry index, and wall thickness), peak stress equations for males and females were developed to predict AAA rupture. According to these simulation results and the two equations developed above, some common and sex-specific features have been revealed.

For both sexes, the geometric features of the AAA have an obvious influence on peak wall stress. Among the three morphological factors, wall thickness is critical for developing a ruptured AAA in both men and women, which suggests that more attention should be paid to its distribution and change when predicting the risk of rupture. Regarding the specific equation for males or females, there were some models where even when the maximum diameter in AAA was relatively small, the corresponding peak wall stress conversely became large, which demonstrated the limitation of maximal transverse measurement as a criterion for AAA rupture risk assessment. In turn, this maximum diameter criterion may even result in delaying AAA repair.

The AAA peak wall stress also has sex differences. When the AAA wall is thin and the asymmetry index is large, or the vessel wall is thick and the asymmetry index is small, the peak wall stress observed in males would be higher than that in females with the same maximum diameter. When the asymmetry index was 0.44 and the corresponding wall thickness was $2 \mathrm{~mm}$, AAAs with maximum diameters of $5 \mathrm{~cm}$ for women and $5.5 \mathrm{~cm}$ for men posed a comparable risk of rupture. This difference should alert surgeons to consider the influence of sex when assessing this risk of rupture of an AAA.

For ease of use in a clinical setting, equations to predict peak wall stress and a $3 \mathrm{D}$ grid have been developed in the present study (Equations 3 and 4, Figure 7). Both equations could assess the risk of AAA rupture in men and women separately. The 3D grid presented in Figure 7 revealed the relationship between peak wall stress in males and females with different maximum diameters, asymmetry index and wall thickness. Thus, the present study provides more biomechanical information with respect to the development and rupture of AAA to help with patient-specific assessment and treatment decision-making, i.e., open surgery or percutaneous deployment of a stent-graft.

As this is a preliminary study, there are several shortcomings. AAA models were simplified and idealised. Numerical calculations used unidirectional fluid-solid coupling. Material properties of the vascular wall were defined as linear, isotropic elastic, and its thickness was assumed to be uniform. These limitations would result in less accurate results than that using more realistic biomechanical properties, e.g., anisotropic, nonlinear, which entails residual stresses and implies statistically distributed heterogeneities ${ }^{[41-43]}$. Despite these simplifications, the results still have qualitative significance. With the development of numerical simulation methodologies and further imaging technology, coupled with 
consideration of blood flow rheology, predictive models of peak wall stress in AAA would be more accurate and complete.

In conclusion, to be more precise and specific in evaluating the risk of rupture in AAA, the present study developed a new prediction method (predictive equations) that included more patient sex and AAA morphology, based on quantitative biomechanical simulation. Results revealed that the risk of an AAA rupture is not only related to morphological factors, but sex differences as well. This study provides a sexspecific clinical reference tool to assess the aforementioned risk. Future studies should integrate more information from simulation and imaging technologies.

\section{DECLARATIONS}

\section{Acknowledgments}

The English was edited and improved by Dominique Fournier, and the authors sincerely thank him profusely for the quality of his contribution and his patience.

\section{Authors' contributions}

Conception and design: Sun AQ, Zhang YX

Data acquisition: Zhang YX

Analysis and interpretation of data: Sun AQ, Guidoin R, Zhang YX

Supervised the work: Sun AQ, Deng XY

Drafted the manuscript: Sun AQ, Zhang YX, Guidoin R, Xu ZP

Sustancially revised the manuscript: Sun AQ, Ren SQ, Zhang YX

Approved the manuscript: Sun AQ, Zhang YX, Xu ZP, Ren SQ, Deng XY, Guidoin R

\section{Availability of data and materials}

Not applicable.

\section{Financial support and sponsorship}

This work was supported by the National Natural Science Foundation of China (No. 11872096, 11772036, 31870940, 11862004), the “111" Project (B13003).

\section{Conflicts of interest}

All authors declared that there are no conflicts of interest.

\section{Ethical approval and consent to participate}

Not applicable.

\section{Consent for publication}

Not applicable.

\section{Copyright}

(c) The Author(s) 2020.

\section{REFERENCES}

1. McPhee JT, Hill JS, Eslami MH. The impact of sex on presentation, therapy and mortality of abdominal aortic aneurysm in the United States 2001-2004. J Vasc Surg 2007;45:891-9.

2. Kontopodis N, Metaxa E, Papaharilaou Y, Tavlas E, Tsetis D, et al. Advancements in identifying biomechanical determinants for abdominal aortic aneurysm rupture. Vascular 2015;23:65-77.

3. Badger SA, Harkin DW, Blair PH, Ellis PK, Kee F, et al. Endovascular repair or open repair for ruptured abdominal aortic aneurysm: a cochrane systematic review. BMJ Open 2016;6:e008391. 
4. Sakalihasan N, Limet R, Defawe OD. Abdominal aortic aneurysm. Lancet 2005;365:1577-89.

5. Powell JT, Gotensparre SM, Sweeting MJ, Brown LC, Fowkes FGR, et al. Rupture rates of small abdominal aortic aneurysms: a systematic review of the literature. Eur J Vasc Endovasc Surg 2011;41:2-10.

6. Zhang N, Fan ZM. Research progress of evaluation on abdominal aortic aneurysm rupture risk with morphological and biomechanical characteristics. Chin J Interventional Imaging Ther 2011;8:443-6. (in Chinese)

7. Raghavan ML, Kratzberg J, Castro de Tolosa EM, Hanaoka MM, Walker P, et al. Regional distribution of wall thickness and failure properties of human abdominal aortic aneurysm. J Biomech 2006;39:3010-6.

8. Vorp DA, Raghavan ML, Webster MW. Mechanical wall stress in abdominal aortic aneurysm: Influence of diameter and asymmetry. J Vasc Surg 1998;27:632-9.

9. Gasser TC, Auer M, Labruto F, Swedenborg J, Roy J. Biomechanical rupture risk assessment of abdominal aortic aneurysms: model complexity versus predictability of finite element simulations. Eur J Vasc Endovasc Surg 2010;40:176-85.

10. Meng H, Tutino VM, Xiang J, Siddiqui A. High WSS or low WSS? Complex interactions of hemodynamics with intracranial aneurysm initiation, growth, and rupture: toward a unifying hypothesis. AJNR Am J Neuroradiol 2014;35:1254-62.

11. Davies PF, Mudel T, Barbee KA. A mechanism for heterogeneous endothelial responses to flow in vivo and in vitro. J Biomech 1995;28:1553-60.

12. Gizzi A, Bernaschi M, Bini D, Cherubini C, Filippi S, et al. Three-band decomposition analysis of wall shear stress in pulsatile flows. Phys Rev E Stat Nonlin Soft Matter Phys 2011;83:031902.

13. Cyron CJ, Wilson JS, Humphrey JD. Mechanobiological stability: a new paradigm to understand the enlargement of aneurysms? J R Soc Interface 2014;11:20140680.

14. Tervahauta M. Long-term outcomes of immediate repair compared with surveillance of small abdominal aortic aneurysms. Acc Current Journal Review 2002;11:1437-44.

15. Lowry D, Singh J, Mytton J, Tiwari A. Sex-related outcome inequalities in endovascular aneurysm repair. Eur J Vasc Endovasc Surg 2016;52:518-25.

16. Mehta M, Byrne WJ, Robinson H, Roddy SP, Paty PS, et al. Women derive less benefit from elective endovascular aneurysm repair than men. J Vasc Surg 2012;55:906-13.

17. De Rango P, Lenti M, Cieri E, Simonte G, Cao P, et al. Association between sex and perioperative mortality following endovascular repair for ruptured abdominal aortic aneurysm. J Vasc Surg 2013;57:1684-92.

18. Deery SE, Soden PA, Zettervall SL, Shean KE, Bodewes TCF, et al. Sex differences in mortality and morbidity following repair of intact abdominal aortic aneurysm. J Vasc Surg 2017;65:1006-13.

19. Powell JT, Brady AR, Brown LC, Fowkes FG, Greenhalgh RM, et al.; The United Kingdom Small Abdominal Aneurysm Trial Participants. Long-term outcomes of immediate repair compared with surveillance of small abdominal aortic aneurysms. N Engl J Med 2002;19:1445-52.

20. Forbes TL, Lawlor DK, DeRose G, Harris KA. Sex differences in relative dilatation of abdominal aortic aneurysms. Ann Vasc Surg 2006;20:564-8.

21. Ash J, Khan KD, Hussain SM. Abdominal aortic aneurysms in women: the debate continues. Endovascular Today 2018;17:51-6.

22. Lo RC, Schermerhorn ML. Abdominal aortic aneurysms in women. J Vasc Surg 2016;63:839-44.

23. Solberg S, Singh K, Wilsgaard T, Jacobsen BK. Increased growth rate of abdominal aortic aneurysms in women. The Tromso stud. Eur J Vasc Endovasc Surg 2005;29:145-9.

24. Dillavou ED, Muluk SC, Makaroun MS. A decade of change in abdominal aortic aneurysm repair in the United States: have we improved outcomes equally between men and women? J Vasc Surg 2006;43:230-8.

25. Chaikof EL, Brewster DC, Dalman RL, Makaroun MS, 1lliq KA, et al. The care of patients with an abdominal aortic aneurysm: the Society for Vascular Surgery practice guidelines. J Vasc Surg 2009;50:S2-49.

26. Ulug P, Sweeting MJ, von Allmen RS, Thompson SG, Powell JT, et al. Morphological suitability for endovascular repair, non-intervention rates, and operative mortality in women and men assessed for intact abdominal aortic aneurysm repair: systematic reviews with metaanalysis. Lancet 2017;389:2482-91.

27. Darling RC, Messina CR, Brewster DC, Ottinger LW. Autopsy study of unoperated abdominal aortic aneurysms. The case for early resection. Circulation 1977;56:II161-4.

28. Doyle BJ, Callanan A, Burke PE, Grace PA, Walsh MT, et al. Vessel asymmetry as an additional diagnostic tool in the assessment of abdominal aortic aneurysms. J Vasc Surg 2009;49:443-54.

29. Di Martino ES, Bohra A, Vande Geest JP, Gupta N, Makaroun MS, et al. Biomechanical properties of ruptured versus electively repaired abdominal aortic aneurysm wall tissue. J Vasc Surg 2006;43:570-6.

30. Wanhainen A, Themudo R, Ahlström H, Lind L, Johansson L. Thoracic and abdominal aortic dimension in 70-year-old men and women-a population-based whole-body magnetic resonance imaging (MRI) study. J Vasc Surg 2008;47:504-12.

31. Scotti CM, Shkolnik AD, Muluk, SC, Finol EA. Fluid-structure interaction in abdominal aortic aneurysms: effects of asymmetry and wall thickness. Biomed Eng Online 2005;4:64.

32. Xu XH, He MZ. Experimental design and design-expert, SPSS Application. Beijing: Science Press; 2010.

33. Olufsen MS, Peskin CS, Kim WY, Pedersen EM, Nadim A. Numerical simulation and experimental validation of blood flow in arteries with structured-tree outflow conditions. Ann Biomed Eng 2000;28:1281-99.

34. Norman PE, Powell JT. Abdominal aortic aneurysm: the prognosis in women is worse than in men. Circulation 2007;115:2865-9.

35. Gatzka CD, Kingwell BA, Cameron JD, Berry KL, Liang YL, et al. Sex differences in the timing of arterial wave reflection beyond 
differences in body height. J Hypertens 2001;19:2197-203.

36. EI Baroudi A, Razafimahery F, Rakotomanana L. Fluid-structure interaction within three-dimensional models of an idealized arterial wall. Int J Eng Sci 2014;84:113-26.

37. Zhao S, Li W, Gu L. Biomechanical prediction of abdominal aortic aneurysm rupture risk: sensitivity analysis. J Biomed Sci 2012;5:664-71.

38. Villard C, Eriksson P, Hanemaaijer R, Lindeman JH, Hultgren R. The composition of collagen in the aneurysm wall of men and women. J Vasc Surg 2017;66:579-85.

39. Vande Geest JP, Dillavou ED, Di Martino ES, Oberdier M, Bohra A, et al. Gender-related differences in the tensile strength of abdominal aortic aneurysm. Ann N Y Acad Sci 2006;1085:400-2.

40. Lindquist Liljeqvist M, Hultgren R, Siika A, Gasser TC, Roy J. Sex, smoking, body size, and aneurysm geometry influence the biomechanical rupture risk of abdominal aortic aneurysms as estimated by finite element analysis. J Vasc Surg 2017;65:1014-21.e4.

41. Nestola MGC, Gizzi A, Cherubini C, Filippi S. Three-band decomposition analysis in multiscale FSI models of abdominal aortic aneurysms. Int J Mod Phys C 2016;27:1650017.

42. Bianchi D, Monaldo E, Gizzi A, Marino M, Filippi S, et al. A FSI computational framework for vascular physiopathology: a novel flowtissue multiscale strategy. Med Eng Phys 2017;47:25-37.

43. Marino M. Constitutive modeling of soft tissues. In: Narayan R, editor. Encyclopedia of biomedical engineering. Elsevier: Oxford, UK; 2019. pp. 81-110. 